\title{
Extractos crudos de Magnolia \\ schiedeana schitdl. para el control de bacterias fitopatógenas
}

\section{Crude extracts of Magnolia schiedeana Schltdl. for the control of phytopathogenic bacteria}

\author{
Thalía Ramírez-Reyes ${ }^{*}$, Norma Flores-Estévez', Mauricio Luna-Rodríguez², Juan Carlos Noa-Carrazana', \\ Lázaro R. Sánchez-Velásquezl y Ángel Trigos-Landa².
}

' Instituto de Biotecnología y Ecología Aplicada. Universidad Veracruzana. Xalapa, Veracruz, México.
${ }^{2}$ Laboratorio de Alta Tecnología de Xalapa. Universidad Veracruzana. Xalapa, Veracruz, México. *Autor de correspondencia. tiramirez1906@gmail.com

\section{RESUMEN}

Se evaluó el efecto bactericida de extractos crudos de diferentes tejidos de Magnolia schiedeana sobre las bacterias fitopatógenas Pectobacterium carotovorum y Pseudomonas cichorii. Los tejidos estudiados fueron polifolículo, semilla, corteza (proveniente de especímenes adultos y de especímenes jóvenes), flor y hoja (proveniente de especímenes adultos y de especímenes jóvenes), utilizando como disolventes acetato de etilo y de etanol. Los efectos bactericidas de los extractos fueron comparados con el efecto de un antibiótico comercial (tetraciclina, $5 \mu \mathrm{g} / \mu \mathrm{l})$. Los extractos que mostraron inhibición bacteriana fueron, el obtenido con acetato de etilo a partir de tejido floral, el cual no igualó la inhibición del antibiótico comercial sobre P. cichorii $(\mathrm{p}<0.001)$ y el extracto etanólico floral, que inhibió el crecimiento de P. carotovorum en la misma proporción que el antibiótico $(\mathrm{p}=0.079)$.

Palabras clave: bacterias fitopatógenas, fenología, magnolia, Pectobacterium carotovorum, Pseudomonas cichorii.

\section{ABSTRACT}

Bactericidal effect was assessed in crude extracts of different tissues of Magnolia schiedeana about the phytopathogenic bacteria Pectobacterium carotovorum and Pseudomonas cichorii. The tissues studied were polifolicule, seed, bark (obtained from adult specimens and young specimens), flower and leave (from both adult and young specimens as well), using as solvents ethyl acetate and ethanol. The extracts that showed bacterial inhibition were obtained one with ethyl acetate from floral tissue, which did not equaled the inhibiting properties of a commercial antibiotic (tetracycline, $5 \mu \mathrm{g} / \mathrm{\mu l}$ ) on P. cichorii $(\mathrm{p}<0.001)$; and the other was floral ethanol extract that inhibited P. carotovorum in the same proportion as the antibiotic $(\mathrm{p}=0.079)$.

KEY wORDS: phytopathogenic bacteria, phenology, magnolia, Pectobacterium carotovorum, Pseudomonas cichorii.

\section{INTRODUCCIÓN}

La seguridad ambiental se ha convertido en una gran preocupación para muchos países debido, entre otros aspectos, al empleo de agroquímicos, los cuales pueden causar daños ambientales severos, tanto al suelo y animales, como a la salud humana, provocando distintos tipos de cánceres, linfomas, asma, dermatitis alérgica, enfermedades respiratorias, malformaciones congénitas, entre otras (Zúñiga et al., 2007; Teng et al., 2010). Aunado a ello, el incremento de multirresistencia en organismos fitopatógenos está impulsando la búsqueda de nuevos agentes biocidas de fuentes alternativas, destacando entre estos a las plantas medicinales (Roy et al., 2010; Vukovic et al., 2007). 
Las magnolias han sido ampliamente utilizadas en la medicina tradicional oriental; especies como Magnolia officinalis, M. obovata, M. grandiflora y M. liliflora presentan actividades antibacterial y antifúngica, por contar con una amplia variedad de compuestos biológicamente activos obtenidos de tejidos como corteza, raíz, hojas, flores y semillas, destacando los fenilpropanoides magnolol y honokiol (Bae et al., 1985; Song y Fischer, 1999; Wang et al., 2000). Pocos estudios han demostrado la capacidad antibacteriana de las especies de magnolia endémicas de México. Recientemente, Jacobo-Salcedo et al. (2011), observaron que extractos etanólicos de semilla de $M$. dealbata presentaron efectos antimicrobianos sobre la bacteria fitopatógena Clavibacter michiganensis subsp. michiganensis, así como en algunas cepas de bacterias y hongos patógenos de humanos.

Otra especie endémica de México es Magnolia schiedeana Schltdl, un árbol perenne y de reproducción anual que habita en el bosque mesófilo de montaña y en el bosque caducifolio; esta especie se restringe a 14 poblaciones dispersas, catalogándose así como especie amenazada (Cicuzza, 2007; Dieringer y Espinoza, 1994; Newton et al., 2008; Vázquez, 1994). Aunque poco estudiada, $M$. schiedeana cuenta con información sobre aspectos ecológicos en torno a su conservación, sin embargo escasos estudios abordan su aprovechamiento en fitosanidad, tal como ocurre con otras especies de magnolia (Jacobo-Salcedo et al., 2011; Mori et al., 1997). No hay información sobre sus propiedades antimicrobianas, ni el estado fenológico de la planta o la posible influencia de los periodos estacionales sobre dichas propiedades.

\section{OBJETIVOS}

El presente trabajo tuvo como objetivo evaluar el efecto antimicrobiano de extractos crudos de diferentes tejidos de individuos jóvenes y adultos de M. schiedeana y el posible efecto de las estaciones del año, sobre el desarrollo in vitro de las bacterias fitopatógenas Pectobacterium carotovorum y Pseudomonas cichorii.

\section{MATERIALES Y MÉTODOS}

\section{Muestras vegetales}

El material vegetal de Magnolia schiedeana se colectó en el Área Natural Protegida de "La Martinica", Banderilla, Veracruz, México $\left(19^{\circ} 35^{\prime} \mathrm{N}, 9^{\circ}\right.$ 56' O). Se evaluó la influencia del estado fenológico sobre la actividad antimicrobiana de extractos crudos, para lo cual se tomaron muestras aleatorias de diez individuos sin estructuras reproductivas, con diámetro a la altura del pecho (DAP) menor a $10 \mathrm{~cm}$ y altura menor a $1.5 \mathrm{~m}$, considerados jóvenes; así como diez individuos que contaron con estructuras reproductivas, DAP mayor a $10 \mathrm{~cm}$ y altura mayor a $1.5 \mathrm{~m}$, considerados adultos. Para evaluar la influencia de los periodos estacionales sobre la actividad antibacteriana, se colectaron muestras de hoja, corteza, flor, polifolículo y semillas iniciando en otoño de 2009 hasta verano de 2010. El muestreo de hojas y corteza se realizó para los dos grupos de edad definidos.

El material vegetal se lavó con agua destilada, se pesó y almacenó a $-20{ }^{\circ} \mathrm{C}$ por 5 días. Posteriormente se liofilizaron con un equipo LABCONCO y se almacenaron a $-4{ }^{\circ} \mathrm{C}$ hasta su utilización.

\section{Preparación de extractos}

El material vegetal liofilizado fue triturado en una licuadora industrial (Fisher, 3BL52). Posteriormente, de cada tejido triturado se prepararon extractos crudos de acetato de etilo y de etanol (25 g de tejido y $250 \mathrm{ml}$ de disolvente), los cuales se maceraron por $72 \mathrm{~h}$, a una agitación de 175 $\min ^{-1}$ (agitador orbital LAB-LINE). Este proceso se realizó en tres ocasiones.

Los extractos se filtraron y se concentraron a presión reducida en un evaporador rotatorio (BUCHI); los extractos fueron almacenados en frascos de vidrio a $-4{ }^{\circ} \mathrm{C}$ hasta su uso.

\section{Pruebas de actividad antibacteriana}

Los organismos de prueba fueron cepas de P. cichorii y $P$. carotovorum, aportadas por el Laboratorio de Alta Tecnología de Xalapa, S. C. (LATEX), Xalapa, Veracruz, 
México. Para su uso, los cultivos bacterianos se activaron en medio B de King a $27^{\circ} \mathrm{C} \pm 2{ }^{\circ} \mathrm{C}$ por 35 h. Para la realización de los bioensayos se utilizaron soluciones bacterianas con $1.5 \times 10^{8} \mathrm{ufc} / \mathrm{ml}$, según la escala de turbidez McFarland (NCCLS, 1994).

La actividad antibacteriana de los extractos vegetales previamente obtenidos, fue evaluada por el método de difusión en disco, como se describe a continuación: Los extractos fueron resuspendidos en una porción suficiente del disolvente correspondiente; posteriormente círculos de papel de filtración media estériles ( $7 \mathrm{~mm}$ de diámetro) fueron impregnados por inmersión en cada extracto (extracto: $35 \mathrm{mg}$, un antibiótico comercial -tetraciclina, $0.325 \mathrm{mg}$-, disolventes acetato de etilo o etanol, y un disolvente como control) y dejados secar sobre un papel aluminio estéril, con la finalidad de que se volatilizara el disolvente empleado. Adicionalmente, $100 \mu \mathrm{l}$ de solución bacteriana mezclada con $18 \mathrm{ml}$ de medio B de King, fueron vaciados en cajas Petri (cinco cajas por tejido estudiado). Posteriormente, en cada caja se colocaron cuatro círculos de papel de filtración grado medio, estériles ( $7 \mathrm{~mm}$ de diámetro), uno impregnado con cada tratamiento. En total fueron cuatro tratamientos por caja, con cinco réplicas por experimento. Las cajas se incubaron a $27{ }^{\circ} \mathrm{C} \pm 2{ }^{\circ} \mathrm{C}$ por $48 \mathrm{~h}$ (Pereira et al., 2006; Vukovic et al., 2007).

\section{Análisis estadísticos}

Para la evaluación de la capacidad antibacteriana, se registraron los diámetros de la zona de inhibición del crecimiento bacteriano alrededor de los círculos de papel. Aquellos tratamientos que no tuvieron efecto fueron eliminados del análisis. Se realizó un análisis de varianza no paramétrico de Friedman, además de comparaciones pareadas para muestras dependientes utilizando la prueba de Wilcoxon entre tratamientos y con respecto a los controles. Para ello se utilizó el software STATISTICA v.8 (StatSoft 2002 Inc. STATISTICA).

\section{Resultados}

Los extractos acéticos y etanólicos obtenidos a partir de flores de M. schiedeana, tejido primaveral propio de árboles adultos, tuvieron un efecto inhibitorio sobre las cepas evaluadas (Tabla 1). El resto de los extractos no mostraron inhibición sobre las bacterias de prueba, no importando el tejido vegetal, estado de desarrollo del árbol, disolvente empleado, ni la temporada del año en que se colectaron dichos materiales.

El extracto floral etanólico inhibió a P. carotovorum en la misma proporción que la tetraciclina ( $\mathrm{p}=0.079)$, mientras que $P$. cichorii no se vio afectada en su crecimiento colonial, en tanto que el extracto floral de acetato

Tabla 1. Actividad antibacteriana de extractos crudos de Magnolia schiedeana sobre las bacterias fitopatógenas Pectobacterium carotovorum y Pseudomonas cichorii.

\begin{tabular}{|c|c|c|c|c|c|c|c|c|c|c|c|c|c|c|}
\hline & \multicolumn{7}{|c|}{ Pectobacterium carotovorum } & \multicolumn{7}{|c|}{ Pseudomonas cichorii } \\
\hline & $\mathbf{P}$ & $\mathrm{S}$ & $\mathrm{CA}$ & C.J. & $\bar{F}$. & HA & HJ. & $\mathbf{P}$ & S. & CA & C.J. & $\bar{F}$. & HA & HJ. \\
\hline & $\mathrm{D} \pm \mathrm{DS}$ & $\mathrm{D} \pm \mathrm{DS}$ & $\mathrm{D} \pm \mathrm{DS}$ & $\mathrm{D} \pm \mathrm{DS}$ & $\mathrm{D} \pm \mathrm{DS}$ & $D \pm D S$ & $\mathrm{D} \pm \mathrm{DS}$ & $D \pm D S$ & $\mathrm{D} \pm \mathrm{DS}$ & $\mathrm{D} \pm \mathrm{DS}$ & $\mathrm{D} \pm \mathrm{DS}$ & $\mathrm{D} \pm \mathrm{DS}$ & $\mathrm{D} \pm \mathrm{SD}$ & $\mathrm{D} \pm \mathrm{DS}$ \\
\hline ACOEt & - & - & - & - & - & - & - & - & - & - & - & $11.35 \pm 1.35$ & - & - \\
\hline Tetraciclina & - & - & - & - & - & - & - & - & - & - & - & $14.6 \pm 0.55$ & - & - \\
\hline $\begin{array}{l}\text { Control } \\
\text { esteritad }\end{array}$ & - & - & - & - & - & - & - & - & - & - & - & - & - & - \\
\hline EtOH & - & - & - & - & $14.95 \pm 0.98$ & - & - & - & - & - & - & - & - & - \\
\hline Tetraciclina & - & - & - & - & $13.6 \pm 0.55$ & - & - & - & - & - & - & - & - & - \\
\hline $\begin{array}{l}\text { Control } \\
\text { esterílad }\end{array}$ & - & - & - & - & - & - & - & - & - & - & - & - & - & - \\
\hline
\end{tabular}

D= diámetros de la zona de inhibición (mm), incluyendo el diámetro del círculo (7 mm), DS= desviación estándar, P= polifolículo, S= semilla, CA= corteza adultos, CJ= corteza jóvenes, $\mathrm{F}=$ flor, $\mathrm{HA}$ = hoja adultos, $\mathrm{HJ}$ = hoja jóvenes, $\mathrm{AcOE}=$ acetato de etilo, $\mathrm{EtOH}=$ etanol.

-No mostró actividad antibacteriana. 
de etilo si bien inhibió el crecimiento de P. cichorii, no logró igualar la capacidad inhibitoria de tetraciclina $(\mathrm{p}<$ 0.001). Sin embargo, este mismo extracto no tuvo efecto inhibitorio frente a P. carotovorum.

\section{DISCUSIÓN}

Las especies pertenecientes al género Magnolia cuentan con una gran variedad de compuestos biológicamente activos, incluyendo lignanos, neolignanos, terpenoides y alcaloides. Estos presentan un amplio espectro de actividad biológica, destacándose su capacidad mosquiticida, antifúngica y antimicrobiana, lo que ha impulsado el estudio para evaluar la actividad de extractos vegetales de diversas especies de este género (Bang et al., 2000; Clark et al.,1981; Kelm et al., 1997).

En este trabajo se observó una inhibición del crecimiento bacteriano de $P$. carotovorum por la acción de extractos etanólicos florales de M. schiedeana; está documentado que extractos etanólicos de diversos tejidos de magnolias han mostrado inhibición sobre diversas bacterias, como C. michiganensis y E. coli, atribuyendo dicha actividad a metabolitos pertenecientes al grupo de los fenoles (Bae et al., 1985; Clark et al., 1981; Jacobo-Salcedo et al., 2011). Se ha determinado la presencia de fenoles en flores de magnolias, como en M. grandiflora, según investigaciones de Huang et al. (2012). Para M. schiedeana no se encontró información sobre los compuestos químicos presentes en flores, por lo que el realizar estudios químicos de esta especie orientará en sus aplicaciones biológicas.

Está ampliamente documentado que aceites esenciales y compuestos fenólicos, obtenidos a partir de extractos de plantas, pueden presentar diversas actividades antimicrobianas, dependiendo de las técnicas de separación y de las polaridades de los disolventes utilizados sobre el mismo extracto vegetal (Bajpai et al., 2008).

Investigaciones relacionadas con el empleo de extractos de diversas especies de magnolias, como M. obovata, M. kobus, M. grandiflora, han mostrado que dependiendo del disolvente es la capacidad de inhibición sobre hongos de importancia agrícola como Pythium aphanidermatum,
R. solani, F. oxysporum, Alternaria alternata, Botrytis cinérea (Ahmed y Abdelgaleil 2005; Choi et al., 2009; Mori et al., 1997). De lo anterior se puede suponer que las magnolias son capaces de presentar actividad biológica en distinta intensidad, ante diversos microorganismos fitopatógenos, con extractos obtenidos a partir de diferentes tejidos y diferentes polaridades de disolventes. De este modo, la actividad antibacteriana observada en este estudio dependió del tejido vegetal y del tipo de disolvente, por lo que es probable que algunos grupos químicos afines a un tipo de disolvente pudieran ser los causantes de la actividad antibacteriana específica por extracto observada.

Para el caso de P. cichorii, no se encontró información de actividad antimicrobiana empleando extractos crudos de magnolias ni de alguna otra especie vegetal, por lo que cabe destacar que haber obtenido actividad biológica empleando extracto de flor con acetato de etilo, representa un elemento promisorio en la búsqueda de agentes antimicrobianos, cuyos resultados podrían ser extrapolados a otros agentes infecciosos ampliamente ubicuos del grupo de las Pseudomonas.

\section{CONCLUSIONES}

Los extractos crudos obtenidos de flores de M. schiedeana poseen compuestos que, diferencialmente al ser extraídos con etanol o acetato de etilo, poseen actividad antimicrobiana ante $P$. carotovorum o $P$. cichorii, por lo que se sugiere determinar los metabolitos secundarios responsables de la actividad biológica observada, ya que puede tratarse de compuestos distintos al magnolol y honokiol, ambos obtenidos con disolventes polares. Esto aporta mayor sustento al estudio de extractos, así como a los compuestos químicos obtenidos de magnolia para el control de bacterias fitopatógenas Gram negativas, con miras a otorgar a esta especie vegetal elementos adicionales para su aprovechamiento y conservación.

\section{RECONOCIMIENTOS}

Al Consejo Nacional de Ciencia y Tecnología (CONACyT) por la beca otorgada para realizar estudios de doctorado (Registro No. 214331). Se Agradece al Laboratorio de 
Alta Tecnología de Xalapa (LATEX) por el uso de sus instalaciones así como a todo el personal que ahí labora.

\section{REFERENCIAS}

Ahmed, S.M. y S.A.M. Abdelgaleil. 2005. Antifungal activity of extracts and sesquiterpene lactones from Magnolia grandiflora L. (Magnoliaceae). International Journal of Agriculture \& Biology 7(4):638-642.

Bae, K., B. Yoo, M. Lee y W. Seo. 1985. Antimicrobial activities of Hydroxybiphenyl Derivatives (I). Archives of Pharmacal Research 8(2):85-89.

Bajpai, V.K., A. Rahman, N.T. Dungs, M.K. Huh y S.C. Kang. 2008. In vitro inhibition of food spoilage and foodborne pathogenic bacteria by essential oil and leaf extracts of Magnolia liliflora Desr. Journal of Food Science 73(6):314-320

Bang, K., Y. Kim, B. Min, M. Na, Y. Rhee, J. Lee y K. Bae. 2000. Antifungal activity of magnolol and honokiol. Archives of Pharmacal Research 23:46-49.

Choi, N.H., G.J. Choi, B.S. Min, K.S. Jang, Y.H., Choi, M.S. Kang, M.S. Park, J.E. Choi, B.K. Bae y J.C. Kim. 2009. Effects of neolignans from the stem bark of Magnolia obovata on plant pathogenic fungi. Journal of Applied Microbiology 106(6):2057-2063.

Cicuzza, D., A. Newton y S.A. Oldfield. 2007. The red list of Magnoliaceae. Fauna and Flora International. 52p.

Clark, A., F. El-Feraly y W. Li. 1981. Antimicrobial activity of phenolic constituents of Magnolia grandiflora L. Journal of Pharmaceutical Sciences 70(8):951-952.

Dieringer, G. y S.J.E. Espinoza. 1994. Reproductive ecology of Magnolia schiedeana (Magnoliaceae), a threatened cloud forest tree species in Veracruz, México. Bulletin of the torrey botanical club 121(2):154-159.

Huang, HC, W.Y. Hsieh, Y.L. Niu y T.M. Chang. 2012.Inhibition of melanogenesis and antioxidant properties of Magnolia grandiflora L. flower extract. BMC Complementary and Alternative Medicine 12: 72.

Jacobo-Salcedo, M. R., L. A. González-Espindola, A. J. AlonsoCastro, M. R. González-Martínez, F. Domínguez y A. García-Carranca. 2011. Antimicrobial activity and cytotoxic effects of Magnolia dealbata and its active com- pounds. Natural Product Communications 6(8):1121-1124.

Kelm, M., M. Nair y R. Schutzki, 1997. Mosquitocidal Compounds from Magnolia salicifolia. International Journal of Pharmacognosy 35(2):84-90.

Mori, M., M. Aoyama y S.Doi. 1997. Antifungal constituents in the bark of Magnolia obovata Thunb. Holz als Rohund Werkstoff 55: 275-278.

NCCLS (National Committee for Clinical Laboratory Standards). 1994. Performance Standards for Antimicrobial Disk and Dilution Susceptibility Test for Bacteria Isolated from Animals; Proposed Standard. NCCLS Document M31-P. National Committee for Clinical Laboratory Standards, Villanova.

Newton, A.C., J. Gow, A. Robertson, G. Williams-Linera, N. Ramírez-Marcial, M. González-Espinosa, T.R. Allnutt y R. Ennos. 2008. Genetic variation in two rare endemic Mexican trees, Magnolia sharpie and Magnolia schiedeana. Silvae Genetica 57(6):348-356.

Pereira, L.S., J.R. Cardoso V., P.L. Medeiros, R.M. Pereira, V.L. Menezes L., H. Satiro X y E. Olivera L. 2006. Antimicrobial activitty of Indigofera suffruticosa. EvidenceBased Complementary and Alternative Medicine 3(2): 261-265.

Roy, S., K. Rao, Ch. Bhuvaneswari, A. Giri y M.L. Narasu. 2010. Phytochemical analysis of Andrographis paniculata extract and its antimicrobial activity. World Journal of Microbiology and Biotechnology 26:85-91.

StatSoft Inc. STATISTICA (Data Analysis Software System). Version 6. 2002. Available at http://www.statsoft.com.

Song, Q. y N.H. Fischer. 1999. Biologically active lignans and neolignans from Magnolia species. Revista de la Sociedad Química de México 43(6):211-218.

Teng, Y., Q. Yang, Z. Yu, G. Zhou, Q. Sun, H. Jin y T. Hou. 2010. In vitro antimicrobial activity of the leaf essential oil of Spiraea alpina Pall. World Journal of Microbiology and Biotechnology 26: 9-14.

Vázquez, J.A. 1994. Magnolia (Magnoliaceae) in Mexico and central America: a synopsis. Brittonia 46(1):1-23.

Vukovic, N., T. Milosevic, S. Sukdolak y S. Solujic S. 2007. Antimicrobial activities of essential oil and methanol 
extract of Teucrium montanum. Evidence-Based Complementary and Alternative Medicine 4(1):17-20.

Wang, S.M., L.J. Lee, Y.T. Huang, J.J. Chen y Y.L. Chen. 2000. Magnolol stimulates steroidogenesis in rat adrenal cells. British Journal of Pharmacology 131:1172-1178.

Zúñiga V., L.A., C.G. Márquez U. y M.S. Duk P. 2007. Estudio citogenético y reproductivo en mujeres temporeras expuestas a pesticidas de la VIII región de Chile. Theoria 16(1):77-87.
Manuscrito recibido el 22 de marzo de 2013.

Aceptado el 31 de agosto de 2014.

Este documento se debe citar como:

Ramírez-Reyes. T., N. Flores-Estévez, M. Luna-Rodríguez, J.C. NoaCarrazana, L.R. Sánchez-Velásquez y Á. Trigos-Landa. 2015. Extractos crudos de Magnolia schiedeana Schltdl. para el control de bacterias fitopatógenas. Madera y Bosques 21(2):159-164. 